Pacific Journal of Mathematics

RIGHT SELF-INJECTIVE RINGS WHOSE ESSENTIAL RIGHT 


\title{
RIGHT SELF-INJECTIVE RINGS WHOSE ESSENTIAL RIGHT IDEALS ARE TWO-SIDED
}

\author{
KenNeth A. Byrd
}

\begin{abstract}
A ring $R$ of the kind described by the title is called a right $q$-ring and is characterized by the property that each of its right ideals is quasi-injective as a right $R$-module. The principal results of this paper are Theorem 6 , which describes how an arbitrary right $q$-ring is constructed from division rings, local rings, and right $q$-rings with no primitive idempotent, and Theorem 5 which shows that a right $q$-ring cannot have an infinite set of orthogonal noncentral idempotents.
\end{abstract}

Ivanov described the structure of indecomposable, nonlocal right $q$-rings and conjectured that every right $q$-ring must be a direct sum of such rings together with a ring all of whose idempotents are central. Our results imply that though the structure of right $q$-rings is slightly more complicated than this (there are chain $q$ rings), one can still reduce the study of $q$-rings to ones which have only central idempotents. More precisely, the study of right $q$ rings is reduced to the study of right self-injective duo rings which are either local or have no primitive idempotent.

The work done here is an extension and generalization of Ivanov's investigations. We develop the finiteness conditions inherent in that work without the assumption of indecomposability and the structure of an arbitrary right $q$-ring is developed at the same time. Throughout the paper all rings have identity $1 \neq 0$ and all modules are unital.

Preliminaries. If one has a decomposition $A=A_{1} \oplus A_{2} \oplus \cdots \oplus A_{n}$ of a right $R$-module $A$ as a finite direct sum of submodules then one has a representation of $\operatorname{End}_{R} A$, the ring of $R$-endomorphisms of $A$, as a ring of $n \times n$ "matrices" of the form $\left(\alpha_{i j}\right)$ where $\alpha_{i j}$ belongs to $\operatorname{Hom}_{R}\left(A_{j}, A_{i}\right)$. In particular, when one has a finite decomposition of the module $R_{R}$ one also has a representation of the ring $R \cong$ End $_{R} R$ as a ring of matrices. A decomposition of $R_{R}=$ $A \oplus B$ as a direct sum of two modules $A$ and $B$ which are unrelated in the sense that $\operatorname{Hom}_{R}(A, B)$ and $\operatorname{Hom}_{R}(B, A)$ are both the trivial group yields a representation of $R$ as the product of the rings $\operatorname{End}_{R} A$ and $\operatorname{End}_{R} B$. For a direct sum decomposition of $R_{R}$, such unrelated summands may be achieved by summing over classes of related summands. When a module $M$ is a direct sum of simple 
modules then a sum over a class of related summands is called an isotypic component of $M$, since two simple modules are related if and only if they are isomorphic.

If $A$ is a right $R$-module then $E(A)$ denotes the injective hull of $A$. When $R$ is right self-injective we will assume that $E(A)$ is a right ideal of $R$ whenever $A$ is a right ideal of $R$. The fact that the rings described by the title are the rings whose right ideals are quasi-injective is a consequence of the fact [6,1.1 Theorem] that $A$ is quasi-injective if and only if $A$ is fully invariant in $E(A)$, that is $\operatorname{End}_{R} E(A) \cdot A \subseteq A$.

Reduction to basic rings. A $q$-ring $R$ will be called basic if each of the nonzero isotypic components of the socle of $R_{R}$ is simple, i.e., $R$ has no two distinct isomorphic minimal right ideals. We shall show that a right $q$-ring is the ring direct sum of a semisimple ring and a basic ring.

The following lemma of [3] is fundamental to our study.

Lemma 1. Let $R$ be a right $q$-ring and $A$ and $B$ be independent right ideals of $R$. If $f$ belongs to $\operatorname{Hom}_{R}(A, B)$ then $f(A)$ is semisimple.

Proof. Recall that the socle of $B$ is the intersection of the essential submodules of $B$. Let $B_{1}$ be an arbitrary essential submodule of $B$. It follows that $A \oplus B_{1}$ is essential in $A \oplus B$. Since $R$ is a right $q$-ring, it follows that $A \oplus B_{1}$ is fully invariant in $A \oplus$ $B$. Letting $g$ be the endomorphism of $A \oplus B$ defined by $g(a+b)=$ $f(a)$ for $a$ in $A$ and $b$ in $B$, we see that $f(A) \subseteq B_{1}$.

COROLlaRy. If $A$ and $B$ are independent isomorphic right ideals of $R$ then each is injective and semisimple. An isotypic component of the socle of $R$ which is not simple is injective.

Proof. Assume that $A$ and $B$ are independent isomorphic right ideals. Since $E(A)$ and $E(B)$ are also independent and isomorphic then the above lemma implies that each is semisimple. It follows that $A=E(A)$ and $B=E(B)$.

Let $H$ be an isotypic component which is not simple. If $H$ is the direct sum of an infinite set of simple modules then $H=H_{1} \oplus H_{2}$ where $H_{1} \simeq H$ and $H_{2} \simeq H$. Since $H_{1}$ and $H_{2}$ are injective then so is $H$. It follows that $H$ must be a finite direct sum of at least two copies of a minimal right ideal $S$ of $R$. Then $S$ is injective and so is $H$. 
Proposition 1. Let $\Gamma$ be an independent set of right ideals of a right q-ring $R$. Suppose that for each member $A$ of $\Gamma$ there is a minimal right ideal $S(A)$ of $R$ so that

(1) if $A \neq B$ then $S(A) \not \equiv S(B)$,

(2) for each $A, \operatorname{Hom}_{R}(A, S(A)) \neq 0$,

(3) $\Sigma\{A \mid A \in \Gamma\} \cap \Sigma\{S(A) \mid A \in \Gamma\}=0$.

Then $\Gamma$ is finite.

Proof. According to (2) there is for each $A$ in $\Gamma$ an epimorphism $\alpha_{A}: A \rightarrow S(A)$ and this induces on the direct sum, the epimorphism $\alpha: \Sigma_{\Gamma} A \rightarrow \Sigma_{\Gamma} S(A)$. Choose hulls in $R$ and extend $\alpha$ to the mapping $\beta: E\left(\Sigma_{\Gamma} A\right) \rightarrow E\left(\Sigma_{\Gamma} S(A)\right)$. From (3) and Lemma 1 we know that the image of $\beta$ is $\Sigma_{\Gamma} S(A)$. On the other hand the image of $\beta$ must be cyclic since $E(\Sigma A)$ is a direct summand of $R$. It follows that there are only finitely many nonisomorphic $S(A)$ for $A$ in $\Gamma$, so (1) implies that $\Gamma$ is finite.

THEOREM 1. A right $q$-ring is isomorphic to the direct product of a semisimple ring and a basic right q-ring.

Proof. The above proposition implies that Soc $R$ has only a finite set $\left\{A_{1}, \cdots, A_{k}\right\}$ of isotypic components which are not simple. Since each of the $A_{i}$ is injective we have a decomposition $R_{R}=\left(\Sigma A_{i}\right) \oplus B$. It follows easily that $R$ is isomorphic to the product of the semisimple ring $\operatorname{End}_{R}\left(\Sigma A_{i}\right)$ and the ring $\operatorname{End}_{R} B$. If $B=e R$ where $e^{2}=e$ then $\operatorname{End}_{R} B \simeq e R e$. Since $e R(1-e) \simeq \operatorname{Hom}_{R}\left(\Sigma A_{i}, B\right)=0$ then $e R e=e R$ so that right ideals of $e R e$ are the same as $R$-submodules of $B$. Then since $B$ has no distinct pair of isomorphic simple submodules, it follows that $e R e$ is basic.

Definition. If $A$ and $B$ are right ideals of $R$ then the notation $A \rightarrow B$ will indicate that $A \cap B=0$ and $\operatorname{Hom}_{R}(A, B) \neq 0$. We shall write $A \rightarrow$ if $A \rightarrow B$ for some $B$, and we shall write $\rightarrow B$ if, for some $A, A \rightarrow B$.

The following finiteness condition is due to Ivanov [3, Lemma 3].

THEOREM 2. Let $R$ be a right q-ring. If $\Gamma$ is an independent set of right ideals of $R$ so that $A \rightarrow$ for each $A$ in $\Gamma$ then $\Gamma$ is finite.

Proof. By Theorem 1 we may assume that $R$ is basic. By Lemma 1 we can find for each $A$ in $\Gamma$ an epimorphism $\alpha_{A}$ from $A$ onto a minimal right ideal $S(A)$ such that $A \cap S(A)=0$. Also, by taking injective hulls, we may assume that each $A$ in $\Gamma$ is a direct 
summand of $R$.

Suppose that $S(A)=S(B)$ for some $B, B$ in $\Gamma$ where $A \neq B$. From the projectivity of $A$ there is a mapping $\beta: A \rightarrow B$ so that $\alpha_{B} \beta=\alpha_{A}$. It follows from Lemma 1 that $\operatorname{Im} \beta$ contains a copy of $S(B)$, so that $S(B) \subseteq B$ since $R$ is basic. This contradiction implies that if $A, B \in \Gamma$ and $A \neq B$ then $S(A) \not \equiv S(B)$.

Let $\Gamma_{1}$ be the set of all $A_{1}$ in $\Gamma$ so that $S\left(A_{1}\right) \subseteq \Sigma\{A \mid A \in \Gamma\}$. Since $R$ is basic and the sum is direct then for each member $A_{1}$ of $\Gamma_{1}$ there is a unique member $\gamma\left(A_{1}\right)$ of $\Gamma$ so that $S\left(A_{1}\right) \subseteq \gamma\left(A_{1}\right)$. We use the mapping $\gamma: \Gamma_{1} \rightarrow \Gamma$ to form the partition $\left\{\gamma^{-1}(A) \mid A \in \operatorname{Im} \gamma\right\}$ of $\Gamma_{1}$. Since $A \notin \gamma^{-1}(A)$ for each $A$ in Im $\gamma$, it follows from Proposition 1 that each member of this partition is a finite set.

Assume that $\Gamma_{1}$ is infinite and let $\phi$ be a function which chooses a member from each nonempty subset of $\Gamma_{1}$. If $X$ is a finite subset of $\Gamma_{1}$ then $X \cup \gamma(X) \cup \gamma^{-1}(X)$ is also finite where $\gamma^{-1}(X)=\cup$ $\left\{\gamma^{-1}(B) \mid B \in X\right\}$ and $\gamma^{-1}(B)=\varnothing$ if $B \notin \operatorname{Im} \gamma$. Denote by $X^{\prime}$ the set complement of $X \cup \gamma(X) \cup \gamma^{-1}(X)$ in $\Gamma_{1}$. We note $X^{\prime} \neq \varnothing$ for all finite subsets $X$ of $\Gamma_{1}$. Define the sequence $\left\{A_{i}\right\}_{i=1}^{\infty}$ in $\Gamma_{1}$ by setting $A_{1}=\phi\left(\Gamma_{1}\right)$ and if $A_{1}, \cdots, A_{n}$ are already chosen then $A_{n+1}=$ $\phi\left(\left\{A_{1}, \cdots, A_{n}\right\}^{\prime}\right)$. Suppose that $\left(\Sigma A_{i}\right) \cap\left(\Sigma S\left(A_{i}\right)\right) \neq 0$. Since $R$ is basic this means that for some $j, k$ one has $\gamma\left(A_{j}\right)=A_{k}$ and this cannot happen by the construction of the sequence. The existence of such a sequence contradicts Proposition 1 so we conclude that $\Gamma_{1}$ is finite.

Since $\Gamma-\Gamma_{1}$ is clearly finite by Proposition 1 then $\Gamma$ is finite.

Injective hulls of minimal right ideals. Let $\mathscr{S}$ be the set of minimal right ideals of a basic right $q$-ring $R$ and let $E(\mathscr{S})=$ $\{E(S) \mid S \in \mathscr{S}\}$ be a chosen set of injective hulls in $R$ for the members of $\mathscr{S}$. For each $S$ in $\mathscr{S}$ there is a primitive idempotent $e_{S}$ of $R$ such that $e_{S} R=E(S)$. According to Lemma 1 if $e$ is a primitive idempotent of $R$ and $\rightarrow e R$ then $e R$ is isomorphic to a member of $E(\mathscr{S})$. In fact if $e R$ is not isomorphic to a member of $E(\mathscr{S})$ then $e$ is central as the next proposition shows.

Proposition 2. Let $e$ be a primitive idempotent of a basic right q-ring $R$. If $e R \rightarrow$, then $\rightarrow e R$.

Proof. Suppose the proposition is false so that $(1-e) \mathrm{Re} \simeq$ $\operatorname{Hom}_{R}(e R,(1-e) R) \neq 0$ but $e R(1-e)=0$. Since $e$ is primitive $e R=$ $e R e$ is a local ring and since $e R(1-e)=0$ then the right ideals of $e R e$ are precisely the $R$-submodules of $e R$. If $J$ is the Jacobson radical of $R$ then $e J$ is the unique maximal right ideal of $e R e$. If $e J=0$ then $e R$ is simple and since $R$ is basic it follows that 
$(1-e) R e=0$ contrary to the assumptions. So $e J \neq 0$ and it follows that $e J$ contains a nonzero cyclic submodule $L$ for which there is a $e R e$-epimorphism $\beta: L \rightarrow e R / e J$ which is also an $R$-epimorphism. The assumption that $(1-e) R e \neq 0$ together with Lemma 1 implies that the simple image $e R / e J$ of $e R$ embeds in $(1-e) R$. Since $(1-e) R$ is injective there is an $R$-homomorphism $\alpha: e R \rightarrow(1-e) R$ so that $\left.\alpha\right|_{L}=\beta$. Since $\operatorname{Im} \alpha$ is semisimple it follows that $\alpha(e J)=0$ so that $\beta=0$ which is a contradiction.

Proposition 3. If $e$ is a primitive idempotent of a basic right $q$-ring $R$ and $\rightarrow e R$, then (1) $S=e R(1-e)$ is a minimal right ideal of $R$, (2) $e R e \simeq \operatorname{End}_{R} S$, and (3) $S$ is the only proper nonzero submodule of $e R$.

Proof. (1) Since $\rightarrow e R$ then $e R(1-e)$ is nonzero and it is contained in the socle of $e R$. Since $e$ is primitive it follows that $e R=$ $E(S)$ for some minimal right ideal $S$ containing $e R(1-e)$. If $\operatorname{Hom}_{R}(e R, S) \neq 0$ then there is a copy of $S$ in $(1-e) R$ contradicting the fact that $R$ is basic. It follows that $s e=0$ for every $s \in S$, that is $S \leqq e R(1-e)$. Thus $S=e R(1-e)$.

(2) If $J$ is the Jacobson radical of $R$ then $e R e$ has radical $e J e=\{x \in e R e \mid x S=0\}$. Since $e R=S \oplus e R e$ as abelian groups one has

$$
(e J e) R=(e J e)(e R) \subseteq(e J e) S+e J e=e J e
$$

so that $e J e$ is a right $R$-submodule of $e R$. Since $S \cap e J e=0$ then $e J e=0$ so that $e R e$ is a division ring. Restriction to $S$ is an isomorphism from $\operatorname{End}_{R} e R$ onto $\operatorname{End}_{R} S$.

(3) If $K$ is a nonzero submodule of $e R$ then $S \subseteq K$ and $K=$ $K e \oplus K(1-e)$. It follows that $S=K(1-e)$. Since $K e$ is a right ideal of $e R e$ then either $K e=0$ or $K e=e R e$. Thus $K=S$ or $K=$ $e R$.

Let $\mathscr{A}(R)=\{E(S) \in E(\mathscr{S}) \mid \rightarrow E(S)\}$. We consider the restriction of the $\rightarrow$-relation to $\mathscr{A}(R)$. Note that $E\left(S_{1}\right) \rightarrow E\left(S_{2}\right)$ for $E\left(S_{1}\right)$, $E\left(S_{2}\right)$ members of $\mathscr{A}$ means that the top, $E\left(S_{1}\right) / S_{1}$, of $E\left(S_{1}\right)$ is isomorphic to the bottom, $S_{2}$, of $E\left(S_{2}\right)$.

Let $D$ be the domain and $T$ be the range of the restriction of $\rightarrow$ to the set $\mathscr{A}$. It is easy to show that $\rightarrow$ is a one-to-one function from $D$ onto $T$. Define $a: \mathscr{A} \rightarrow \mathscr{A}$ by $a\left(E_{1}\right)=E_{2}$ if $E_{1} \in D$ and $E_{1} \rightarrow E_{2}$ and $a\left(E_{1}\right)=E_{1}$ if $E_{1} \notin D$. Similarly $a^{-1}: \mathscr{A} \rightarrow \mathscr{A}$ is defined by $a^{-1}\left(E_{2}\right)=E_{1}$ if $E_{2} \in R$ and $E_{1} \rightarrow E_{2}$ and $a^{-1}\left(E_{2}\right)=E_{2}$ if $E_{2} \notin T$. Then for each $E \in \mathscr{A}$ let $\vec{E}=\left\{a^{k}(E) \mid k \in Z\right\}$. It is easy to see that (1) $E \in \vec{E}$ since $a^{0}$ is the identity mapping, and (2) if $F \in \vec{E}$ then $\vec{F}=$ 
$\vec{E}$ so that the set $\overrightarrow{\mathscr{A}}$ of $\rightarrow-$ classes $\vec{E}$ for $E \in \mathscr{A}$ is a partition of $\mathscr{A}$. In fact the associated equivalence relation on $\mathscr{A}$ is just the smallest equivalence relation on $\mathscr{A}$ which contains the restriction of $\rightarrow$ to $\mathscr{A}$.

It is immediate from Theorem 2 that the set of classes $\vec{E}$ with more than one member is a finite set and also that each class $\vec{E}$ is itself finite. It is straightforward to show that these classes $\vec{E}$ are of two kinds namely;

(1) Chain: $\rightarrow E_{1} \rightarrow E_{2} \rightarrow \cdots E_{l}$ where $E_{1} \notin T$ and $E_{l} \notin D$.

(2) Loop: $E_{1} \rightarrow E_{2} \rightarrow \cdots \rightarrow E_{l} \rightarrow E_{1}$.

In each case the cardinality $l$ of $\vec{E}$ will be called the length of $\vec{E}$.

LEMMA 2. Suppose that $e$ is a primitive idempotent of a basic right $q$-ring $R$ and $\rightarrow e R$ so that $S=e R(1-e) \neq 0$.

(1) The right annihilator $S^{r}=\{x \in R \mid S x=0\}$ is a maximal right ideal.

(2) If $f$ is also a primitive idempotent of $R$ and $e R \rightarrow f R$ then $e R e \simeq f R f$.

Proof. (1) If $s$ is a nonzero element of $S$ then $s^{r}=M$ is a maximal right ideal. The right ideal $M$ must be essential since otherwise $S=e R$ and since $R$ is basic this contradicts the assumption $e R(1-e) \neq 0$. It follows that $M$ is a two-sided ideal of $R$. For any nonzero element $s_{1}$ of $S$ one has $s_{1}=s r$ for some $r$ in $R$ so $s_{1} M=s r M \subseteq s M=0$. Thus $M=S^{r}$.

(2) Let $T$ be the simple submodule of $f R$. Since $T=f R e$ is a simple right $R$-module, it is a 1-dimensional $e R e$-space on the right. Jacobson's density theorem [5, p. 28] and (1) imply that $T$ is also a 1-dimensional $f R f$-space on the left. Choose a nonzero element $t$ of $T$. The correspondence $a \leftrightarrow b$ if and only if $a t=t b$ is an isomorphism between $f R f$ and $e R e$.

The $\rightarrow$-classes $\vec{E}$ of $R$ are determined "up to isomorphism" by our choice of a representative set of injective hulls of minimal right ideals of $R$. However, the sum of an $\rightarrow$-class is independent of this choice. This is a consequence of the following proposition.

Proposition 4. Let $e$ be an idempotent of the basic right $q$ ring $R$. There is a one-to-one correspondence between the set $(1-e) R e$ and the set of copies of $e R$ in $R$ such that to the element $z$ of $(1-e)$ Re corresponds the module $(1+z) e R$.

Proof. If $z$ belongs to $(1-e) R e$ then $f=(1+z) e$ is idempotent and since $e f=e$ and $f e=f$ it follows that $f R \simeq e R$. If $\left(1+z_{1}\right) e R=$ 
$\left(1+z_{2}\right) e R$ for $z_{1}, z_{2}$ in $(1-e) R e$ then for some $r$ in $R,\left(1+z_{1}\right) e=$ $\left(1+z_{2}\right) e r$. It follows that $e=e r$ and $z_{1}=z_{1} e=z_{2} e=z_{2}$. Thus the correspondence is one-to-one.

Let $E$ be a copy of $e R$ in $R$. Since $(1-e) R$ contains no nonzero copy of a submodule of $e R$ then the kernel, $E \cap(1-e) R$, of the projection $x \mapsto e x$ of $E$ into $e R$ is zero. It follows that $e R=e E \oplus$ $A$ for some submodule $A$ of $e R$. But $A$ must be zero since there is a copy of $A$ in $e E$. Thus the projection of $E$ into $e R$ is an isomorphism onto $e R$. Choose $a$ in $E$ so that $e a=e$ and let $z=$ $(1-e) a e$. If $x \in E$ then $e(a e x-x)=0$ and it follows that $a e x=x$ for all $x$ in $E$. Then for $x$ in $E$ one has

$$
x=e x+(1-e) x=e x+(1-e) a e x=e x+z x=(1+z) e x .
$$

Thus one has $E=(1+z) e R$.

In particular if $e R \rightarrow f R$ with $e R$ and $f R$ members of $\mathscr{A}$ then every copy of $e R$ in $R$ is contained in $e R \oplus f R$ because $(1-e) R e=$ $f R e$. Thus the sum of an $\rightarrow$-class is independent of the choice of the injective hulls.

Definition. A basic right $q$-ring $R$ is called a loop $q$-ring if $R$ has only one $\rightarrow$-class, that class is a loop, and $R$ is the sum of its loop.

Notation. Let $D$ be a division ring. We denote by $D_{0}$ the $D-D$ bimodule $D$ equipped with the zero multiplication.

THEOREM 3. If $R$ is a loop q-ring of length $l$ then there is a division ring $D$ so that $R$ is isomorphic to the ring $H(l, D)$ of $l \times$ $l$ matrices with elements on the diagonal from $D$ and elements in the positions $(2,1),(3,2), \cdots,(l, l-1),(1, l)$ from $D_{0}$ and zero entries elsewhere. Conversely every ring $H(l, D)$ is a loop q-ring.

Proof. The first statement is an immediate consequence of the matrix representation of $R=\operatorname{End}_{R} R$ where $R_{R}=\sum_{i=1}^{l} E_{i}$ and $E_{1} \rightarrow$ $E_{2} \rightarrow \cdots \rightarrow E_{l} \rightarrow E_{1}$. One may take $D=\operatorname{End}_{R} E_{1}$ and use Lemma 2 (2). The converse is proved in [3, Theorem 3].

The following theorem may be proved by a straightforward induction on the number of loops of $R$.

THEOREM 4. Let $R$ be a basic right q-ring. There is a set $\left\{l_{1}, l_{2}, \cdots, l_{k}\right\}$ of integers $\geqq 2$ and a set of division rings $\left\{D_{1}\right.$, 
$D_{2}, \cdots, D_{k}$ s so that

$$
R \cong \prod_{i=1}^{k} H\left(l_{i}, D_{i}\right) \times R_{1}
$$

where $R_{i}$ is a basic right q-ring which has no loops.

Chain $q$-rings. Assume that $R$ is a basic right $q$-ring with no loops. Suppose that $\mathscr{C}=\left\{\vec{E}_{i} \mid 1 \leqq i \leqq m\right\}$ is a finite set of chains of $R$ where $\vec{E}_{i}$ is $\rightarrow E_{i 1} \rightarrow E_{i 2} \rightarrow \cdots \rightarrow E_{i l_{i}}$ with $E_{i j}=e_{i j} R$ for a primitive idempotent $e_{i j}$ of $R$. Let $f=1-\Sigma e_{i j}$. Then for each $i$ one has $f R \rightarrow E_{i j}$ exactly when $j=1$. Also since $f R(1-f)=0$ then $f R=f R f$ is a ring with identity $f$.

Proposition 5. With the notation above, the ring $f R$ is a basic right q-ring. The set of arrow classes of $R$ is the disjoint union of the set of arrow classes of $f R$ with the set $\mathscr{C}$. For each $i$, the $f R$-module $e_{i 1} R f$ is simple, injective and is not embeddable in $f R$.

Proof. The first two statements are straightforward consequences of the facts that the right ideals of $f R$ coincide with the $R$-submodules of $f R$ and $\operatorname{Hom}_{f R}(K, L)=\operatorname{Hom}_{R}(K, L)$ for any right ideals $K$ and $L$ of $R$ on which $f$ acts as a right identity. Since $e_{i 1} R f$ is a simple $R$-module it is a simple $f R$-module and as $R$ is basic it cannot be isomorphic to a right ideal of $f R$. The $f R$-injectivity of $e_{i 1} R f$ follows from Baer's criterion and Lemma 1.

Suppose that $f R=g R+h R$ where $g$ and $h$ are orthogonal idempotents of $f R$. For each $\vec{E}_{i}$ in $\mathscr{C}$ exactly one of $g R \rightarrow E_{i 1}$ or $h R \rightarrow E_{i 1}$ is true because if both $g R$ and $h R$ mapped onto the simple submodule of $E_{i 1}$ then projectivity of $g R$ would imply that $h R$ contained a copy of that simple module thus violating the agreement that $R$ is basic. If, say, $g R \rightarrow E_{i 1}$ we say the chain $\vec{E}_{i}$ is associated with $g R$. In this way each decomposition of $f$ as a sum of orthogonal idempotents induces a corresponding partition of the set of chains $\mathscr{C}$. The proof of the next proposition describes a procedure for decomposing $f$ in such a way that each component summand of $f R$ has associated with it exactly one chain from $\mathscr{C}$.

Proposition 6. Let $\Lambda$ be an independent set of right ideals of a right q-ring $R$. If there is a right ideal $A$ of $R$ such that (1) $A \rightarrow B$ for every $B \in \Lambda$ and $(2) A \cap(\Sigma B)=0$, then $\Lambda$ is finite.

Proof. We may assume that $R$ is basic, that the members of 
$\Lambda$ are minimal right ideals and that $A=e R$ for some idempotent $e$ of $R$.

Suppose that $B_{1}$ and $B_{2}$ belong to $A$ and $B_{1} \neq B_{2}$. Since $R$ is basic $B_{1} \not B_{2}$ and it follows from Lemma 2(1) that $B_{1}^{r} \neq B_{2}^{r}$. From $e R \rightarrow B$ for each $B \in \Lambda$ it follows that $B e \neq 0$. In particular $e \notin B_{i}^{r}$ for $i=1,2$ and since $R / B_{i}^{r}$ is a division ring it follows that $1-e \epsilon$ $B_{1}^{r} \cap B_{2}^{r}$. The modular law implies that

$$
B_{i}^{r}=(1-e) R+\left(B_{i}^{r} \cap e R\right)
$$

and

$$
\begin{aligned}
B_{i}^{r} \cap e R & =B_{i}^{r} \cap(e R e+e R(1-e)) \\
& =e R(1-e)+\left(B_{i}^{r} \cap e R e\right) .
\end{aligned}
$$

Thus if $B_{1} \neq B_{2}$ then $e R e \cap B_{1}^{r} \neq e R e \cap B_{2}^{r}$.

Choose $x \in\left(e R e \cap B_{1}^{r}\right)-B_{2}^{r}$. Let $J$ denote the Jacobson radical of $R$. Since by [1, Theorem 3.1] eRe/eJe is a regular ring there is an element $y$ of $e R e$ such that $x-x y x$ belongs to $e J e$. Since idempotents of $e R e$ lift modulo eJe by [1, Theorem 4.1] then there is an idempotent $g$ of $e R e$ such that $x y-g$ belongs to eJe. We note that $g \in B_{1}^{r}-B_{2}^{r}$. Thus one has the decomposition $A=(e-g) R \oplus g R$ where $g R \rightarrow B_{2}$ and $(e-g) R \rightarrow B_{1}$.

Assume that $A$ is infinite. Choose one of $g R$ and $(e-g) R$ which has infinitely many members of $\Lambda$ as homomorphic images and call it $A_{1}^{\prime}$ and call the other $A_{1}$ so that $A=A_{1} \oplus A_{1}^{\prime}$. Replace $A$ by $A_{1}^{\prime}$ and repeat the above process so that $A_{1}^{\prime}=A_{2} \oplus A_{2}^{\prime}$ where $A_{2}^{\prime}$ has infinitely many homomorphic images in $A$. In this way we construct an infinite sequence $\left\{A_{i}\right\}_{i=1}^{\infty}$ which satisfies the three conditions of Proposition 1 and this is a contradiction.

Definition. A basic right $q$-ring $R$ is called a chain $q$-ring if $R=f R \oplus E_{1} \oplus \cdots \oplus E_{l}$ where $\rightarrow E_{1} \rightarrow E_{2} \rightarrow \cdots \rightarrow E_{l}$ is the only $\rightarrow$-class of $R$ and $f R \rightarrow E_{1}$. We call $f R$ the corner of $R$ in this case.

Note that in a chain $q$-ring $f R$ is a basic right $q$-ring all of whose idempotents are central since $f R$ has no $\rightarrow$-classes. Also $f R$ is not a right cogenerator since the simple module $E_{1} f$ does not embed in $f R$. For instance $f R$ might be an infinite product of division rings.

Proposition 7. If $R$ is a basic loopless right q-ring then $R$ is isomorphic to the product of a finite set of chain q-rings each of which has as a corner an infinite product of division rings together with a basic loopless q-ring which has no projective minimal right 
ideal.

Proof. In the basic loopless $q$-ring $R$ let $\left\{e_{i} R \mid i \in I\right\}$ be the set of projective minimal right ideals. For each $i$ in $I$ one has $e_{i} R(1-$ $\left.e_{i}\right)=0$ so that $e_{i} R=e_{i} R e_{i}$ is a division ring. Consider the usual embedding $\alpha$ of the direct sum $\sum e_{i} R$ into $\Pi e_{i} R$ where $\alpha$ maps $e_{j}$ to $\left(\delta_{i j} e_{i}\right)_{i \in I}$. Since $e_{i} R=e_{i} R e_{i}$ and $e_{i} R e_{j}=0$ for $i \neq j$ then $\alpha$ is an essential embedding. It follows that there is an $R$-monomorphism $\phi: \Pi e_{i} R \rightarrow R$ so that $\phi \cdot \alpha$ is the inclusion of $\Sigma e_{i} R$ in $R$. Let $\psi$ be the splitting map for $\phi$ so $\psi \phi=1$. One may show that $\psi(1)=\left(e_{i}\right)_{i \in I}$. If $g=\phi \psi(1)$ then the image of $\phi$ is $g R$ and $\psi(g)=\left(e_{i}\right)_{i \in I}$. Since $R$ is basic, $g R(1-g)=0$ so $g R=g R g$. One has for $r, s$ in $R$

$$
\psi(g r \cdot g s)=\psi(g r s)=\psi(g) r s=\left(e_{i}\right)_{i \in I} r s=\left(e_{\imath} r s\right)_{i \in I}=\left(e_{\imath} r\right)_{i \in I} \cdot\left(e_{i} s\right)_{i \in I}
$$

where the last multiplication is componentwise. Thus $\phi$ is a ring isomorphism from $\Pi e_{i} R$ onto $g R$.

Proposition 6 implies that the set of chains $\mathscr{C}(g R)$ of $R$ associated with $g R$ is finite, and that there is a decomposition $g=g_{1}+$ $g_{2}+\cdots+g_{k}$ so that the $g_{i}$ are orthogonal idempotents associated one-to-one with the chains of $\mathscr{C}(g R)$, i.e., each $\mathscr{C}\left(g_{i} R\right)$ is a singleton. Let $\bar{g}_{i}$ be an idempotent such that $\bar{g}_{i} R=g_{i} R \oplus \Sigma \mathscr{C}\left(g_{i} R\right)$. One checks that for each $i=1, \cdots, k, \bar{g}_{i}$ is central. For instance $\bar{g}_{i} R\left(1-\bar{g}_{i}\right)=$ 0 since otherwise $\left(1-\bar{g}_{i}\right) R$ has a simple image in $\bar{g}_{i} R$ and by projectivity must contain a copy of that simple module, thus contradicting the fact that $R$ is basic. Thus $R=\bar{g} R \oplus(1-\bar{g}) R$ where $\bar{g}=$ $\sum_{1}^{k} \bar{g}_{i}$ and each $\bar{g}_{i}$ is central. For each $i, \bar{g}_{i} R$ is a chain $q$-ring with corner, $g_{i} R$, a product of division rings. Also $(1-\bar{g}) R$ is a basic loopless $q$-ring which has no projective minimal right ideal since any such must be contained in $\bar{g} R$ by construction.

Matrix representation of chain $q$-rings. A chain $q$-ring $R$ is a $q$-ring with orthogonal idempotents $f, e_{1}, e_{2}, \cdots, e_{l}$ such that the $e_{i}, 1 \leqq i \leqq l$, are primitive, $f R \rightarrow e_{1} R \rightarrow e_{2} R \rightarrow \cdots \rightarrow e_{l} R, f R=f R f$, and $R=f R \oplus e_{1} R \oplus \cdots \oplus e_{l} R$. Since the $\rightarrow$-relations shown are the only ones which exist between the modules $f R, e_{1} R, \cdots, e_{l} R$ one has the matrix representation

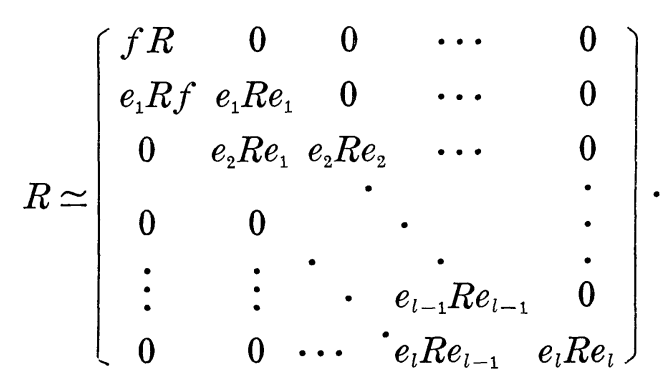


Since $e_{1} R f$, is 1-dimensional as a left $e_{1} R e_{1}$-space if we select $x_{1} \in e_{1} R f, x_{1} \neq 0$ and if $M=x_{1}^{r}$ then $d_{1} x_{1}=x_{1} \bar{d}_{1}$ implements a ring isomorphism $d_{1} \mapsto \bar{d}_{1}$ from $e_{1} R e_{1}$ onto $f R / M$ and at the same time $e_{1} R f \simeq f R / M$ as an $f R$-module. If we use these isomorphisms to identify $e_{1} R e_{1}$ with $f R / M$ and $e_{1} R f$ with the $f R$-module $f R / M$ then the left action of $e_{1} R e_{1}$ on $e_{1} R f$ corresponds to the natural left $f R /$ $M$-module structure of $f R / M$. Similarly for $i \geqq 1$ each $e_{i+1} R e_{i}$ is 1 -dimensional on each side so that selecting $x_{i+1} \in e_{i+1} R e_{i}, x_{i+1} \neq 0$ we have isomorphisms $d_{i+1} \mapsto \overline{d_{i+1}}$ from $e_{i+1} R e_{i+1}$ onto $e_{i} R e_{i}$ given by $d_{i+1} x_{i+1}=x_{i+1} \bar{d} \overline{i+1}$. If we denote by $(f R / M)_{0}$ the abelian group of $f R / M$ with its usual left and right module structures over the rings $f R$ and $f R / M$ and with the zero multiplication then it is easy to see that

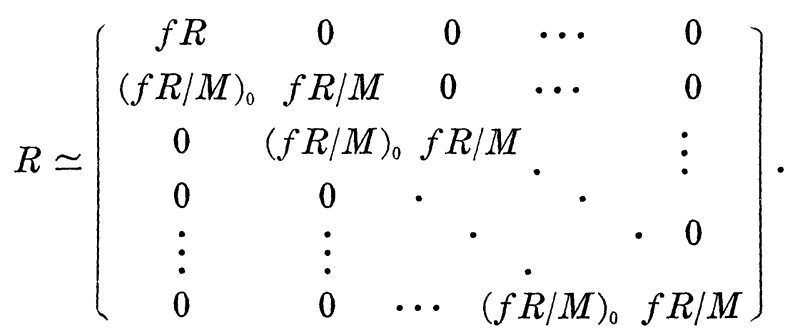

The following proposition shows that, conversely, every ring of this form is a right $q$-ring.

Definition. Let $A$ be a right $q$-ring with an essential maximal right ideal $M$ such that $A / M$ is injective and does not embed in $A$. We denote by $C(A, M, l)$ the ring of $(l+1) \times(l+1)$ matrices with entries in the $(1,1)$ position from $A$, entries in the other main diagonal positions from $A / M$, entries on the sub-diagonal from $(A / M)_{0}$, and zero entries elsewhere. (It is convenient to allow $l$ to be any integer $\geqq 0$.)

Proposition 8. For any $l \geqq 0$, the ring $C(A, M, l)$ as defined above is a right q-ring.

Proof. Let $A$ and $M$ be as described above. For each $l \geqq 1$ let $A_{l}=C(A, M, l)$ and let $M_{l}$ denote the ideal of $A_{l}$ whose members are those matrices with zero entry in the $(l+1, l+1)$ position. We wish to show by induction that for every $l \geqq 1$ the ring $A_{l}$ is a right $q$-ring with the essential maximal right ideal $M_{l}$ such that $A_{l} / M_{l}$ is $A_{l}$-injective and does not embed in $A_{l}$.

If $l \geqq 1$ there is an obvious ring isomorphism between $A_{l+1}$ and $C\left(A_{l}, M_{l}, 1\right)$. Using this, the proof by induction is reduced to prov- 
ing that the statement holds when $l=1$.

Let $e_{i}, i=1,2$ be the idempotent matrix of $A_{1}$ with zero entries except at the $(i, i)$ position where the entry is 1 . Since $e_{1} A_{1} e_{2}=0$ then a minimal right ideal of $A_{1}$ is either a minimal right ideal of $e_{1} A_{1}$ (i.e., a minimal right ideal of $A$ ) or it is a simple submodule of $e_{2} A_{1}$. The kernel of the ring homomorphism from $A_{1}$ onto $A / M$ which sends a matrix $X$ to $X e_{2}$ is $M_{1}=A_{1} e_{1}$. Since $A / M$ is a division ring, the ideal $M_{1}$ is a maximal right ideal of $A_{1}$. It is easy to check that $S=e_{2} A_{1} e_{1}$ is an essential submodule of $e_{2} A_{1}$ so that $M_{1}$ is an essential right ideal of $A_{1}$. Since $e_{2} A_{1} / S \simeq A_{1} / M_{1}$ it follows that $S$ is the only proper nonzero submodule of $e_{2} A_{1}$.

Suppose that $K$ is an essential right ideal of $A_{1}$. If $K \supseteqq e_{2} A_{1}$ then $K=e_{2} A_{1} \oplus\left(K \cap e_{1} A_{1}\right)$. Otherwise $K \cap e_{2} A_{1}=S$ so $K e_{2}=0$ and $K \subseteq M_{1}=e_{1} A_{1} \oplus S$. It follows that $K=S \oplus\left(K \cap e_{1} A_{1}\right)$. Since $A$ is a right $q$-ring it is easy to see that $K \cap e_{1} \mathrm{~A}_{1}$ is a two-sided ideal of $e_{1} A_{1}$ and it follows easily that in either of the above cases, $K$ is a two-sided ideal of $A_{1}$.

To see that $A_{1}$ is right self-injective it suffices to apply Baer's criterion as follows. Let $\phi: K \rightarrow A_{1}$ be an $A_{1}$-homomorphism where $K$ is an essential right ideal of $A_{1}$. Since $K$ is an ideal $K=e_{1} K \oplus$ $e_{2} K$. Let $\phi_{1}$ be the restriction of $\phi$ to $e_{1} K$. Since $e_{1} A_{1}=e_{1} A_{1} e_{1}$ then $\operatorname{Im} \phi_{1} \subseteq A_{1} e_{1}=e_{1} A_{1} \oplus S$. The injectivity of $A / M$ as an $A$-module implies that $S$ is an injective $e_{1} A_{1}$-module and by assumption $e_{1} A_{1} \simeq$ $A$ is right self-injective. Since $\phi_{1}$ is an $e_{1} A_{1}$-homomorphism it follows that there is an element $a$ of $A$ and an element $s$ of $A / M$ so that for every $X$ in $e_{1} K$ one has $\phi_{1}(X)=\left(\begin{array}{ll}a & 0 \\ s & 0\end{array}\right) \cdot X$. Let $\phi_{2}$ be the restriction of $\phi$ to $e_{2} K$. Since no submodule of $e_{2} A_{1}$ has a nonzero image in $e_{1} A_{1}$ then the image of $\phi_{2}$ must be contained in $e_{2} A_{1}$. It is then easy to see that there is an element $d$ of $A / M$ so that for each $Y$ in $e_{2} K$ one has $\dot{\phi}_{2}(Y)=\left(\begin{array}{ll}0 & 0 \\ 0 & d\end{array}\right) \cdot Y$. It follows that for all $Z$ in $K$, $\dot{\phi}(Z)=\left(\begin{array}{ll}a & 0 \\ s & d\end{array}\right) \cdot Z$. So $A_{1}$ is right self-injective.

If $A_{1} / M_{1}$ embeds in $A_{1}$ then either $A_{1} / M_{1}$ embeds in $e_{1} A_{1}$ or $A_{1} / M_{1} \simeq S$. Since $A_{1} / M_{1} \simeq e_{2} A_{1} / S$ and $e_{1} A_{1} e_{2}=0$ then $A_{1} / M_{1}$ does not embed in $e_{1} A_{1}$. If $A_{1} / M_{1} \simeq S$ then there is an epimorphism from $e_{2} A_{1}$ onto $S$. But since $S e_{2}=0$ there is no such mapping. Thus $A_{1} / M_{1}$ does not embed in $A_{1}$. To see that $A_{1} / M_{1}$ is $A_{1}$-injective suppose that $\phi: K \rightarrow A_{1} / M_{1}$ is an epimorphism where $K$ is an essential right ideal of $A_{1}$. Since $K$ is an ideal then $K=e_{1} K \oplus e_{2} K$. Because $e_{1} A_{1} e_{2}=0$ and $A_{1} / M_{1}$ is an image of $e_{2} A_{1}$ it follows that $\dot{\phi}\left(e_{1} K\right)=0$. If $e_{2} K=e_{2} K_{1}$ then $\dot{\phi}$ extends immediately to $A_{1}$. Otherwise, $e_{2} K=S$ so that $\phi$ is an isomorphism between $S$ and $A_{1} / M_{1}$ which we have just shown to be impossible. 
The finiteness condition. The finiteness results Propositions 1 and 6 and Theorem 2 will be subsumed in the following theorem whose proof will be given as a sequence of lemmas.

THEOREM 5. A right q-ring has no infinite set of orthogonal, noncentral idempotents.

It suffices to prove the result for basic rings where from Lemma 1 and Proposition 2, the theorem is equivalent to the assertion that $\mathscr{A}(R)$ is a finite set. From Theorem 4 and Proposition 7 we may assume $R$ has no loops and no projective minimal right ideals. We now reduce the problem to the case where $R$ has no chain of length $l>1$. Let $\left\{\vec{E}_{i} \mid 1 \leqq i \leqq m\right\}$ be the set of chains of $R$ of length $l>1$, where $\vec{E}_{i}$ is $\rightarrow E_{i 1} \rightarrow E_{i 2} \rightarrow \cdots \rightarrow E_{i l_{i}}$ with $E_{i j}=e_{i j} R$ for a primitive idempotent $e_{i j}$ of $R$. Let $f=1-\Sigma e_{i j}$ so that $f R \rightarrow E_{i 1}$ for each $i$ and $f R=f R f$. It follows that $f R$ is a right $q$-ring which is basic, loopless, without projective minimal right ideals and whose $\rightarrow$-classes are exactly the chains of $R$ of length 1 .

LEMMA 3. Let $R$ be a basic right q-ring whose only $\rightarrow$-classes are chains of length 1 and let $\mathscr{S}$ be the set of minimal right ideals of $R$. To each subset $A$ of $\mathscr{S}$ we associate an idempotent $e_{A}$ so that $e_{A} R$ is an injective hull of $\Sigma\{S \mid S \in A\}$. If $A \cong \mathscr{S}$ then there is a subset $A_{1}$ of $\mathscr{S}$ so that $A \Delta A_{1}$ is finite and $e_{A_{1}}$ is central. (Here $\triangle$ denotes symmetric difference of sets.)

Proof. Let $A \subseteq \mathscr{S}$ and $e_{A} R$ be a hull of $\Sigma\{S \mid S \in A\}$. Let $B \cong$ $\mathscr{S}$ be the finite set of simple images of $e_{A} R$ in $\left(1-e_{A}\right) R$ and let $C \subseteq \mathscr{S}$ be the finite set of simple images of $\left(1-e_{A}\right) R$ in $e_{A} R$. One has $e_{A} R=e R+e_{C} R$ and $\left(1-e_{A}\right) R=f R+e_{B} R$ where $e, f, e_{C}$ and $e_{B}$ are pairwise orthogonal idempotents. If $A_{1}=(A-C) \cup B$ then $A \Delta A_{1}$ is finite and $\left(e+e_{B}\right) R$ is a hull of $\Sigma\left\{S \mid S \in A_{1}\right\}$. It is routine to check that $e+e_{B}$ is central. For instance, to see that $\operatorname{Hom}_{R}((1-e-$ $\left.\left.e_{B}\right) R, \quad\left(e+e_{B}\right) R\right)=\operatorname{Hom}_{R}\left(f R+e_{C} R, e R+e_{B} R\right)=0$ one argues as follows: Any simple submodule of $e_{B} R$ is an image of $e_{A} R$. It cannot also be an image of $\left(1-e_{A}\right) R$ so it cannot be an image of $f R$ and thus $\operatorname{Hom}_{R}\left(f R, e_{B} R\right)=0$. Since the $\rightarrow$-classes are all chains of length one then $\operatorname{Hom}_{R}\left(e_{C} R, e R\right)=\operatorname{Hom}_{R}\left(e_{C} R, e_{B} R\right)=0$ and $\operatorname{Hom}_{R}(f R$, $e R)=0$ by the definition of $C$. The argument that $\operatorname{Hom}_{R}\left(\left(e+e_{B}\right) R\right.$, $\left.\left(1-e-e_{B}\right) R\right)=0$ is similar.

Proof of Theorem 5. The proof is by contradiction. Assume the theorem is false. Then there is a right $q$-ring $R$ such that 
$\mathscr{S}=\left\{S_{i} \mid i \in I\right\}$, the set of minimal right ideals of $R$, is infinite, $S_{i} \not$ $S_{j}$ for $i \neq j$, and if for each $i$ we let $e_{j} R$ be a hull of $S_{i}$ then $\left(1-e_{i}\right) R e_{i}=0$ and $e_{i} R\left(1-e_{i}\right) \neq 0$. If we let $A$ be a countably infinite subset of $\mathscr{S}$ then by the Lemma 3 there is a countably infinite subset $A_{1}$ so that the hull of $\Sigma\left\{S \mid S \in A_{1}\right\}$ is generated by a central idempotent $e_{A_{1}}$. It follows that we can assume that $\mathscr{S}$ is countable (so we take $I$ to be the set of positive integers) and that $R_{R}$ is the hull of $\sum_{i=1}^{\infty} S_{i}$, i.e., the socle of $R_{R}$ is essential in $R_{R}$. The proof is given as a sequence of eleven assertions proved individually.

(1) If $J$ is the Jacobson radical of $R$ then

$$
J=\left\{r \in R \mid r e_{i}=0, \forall i\right\}=\bigcap_{i=1}^{\infty} R\left(1-e_{i}\right) .
$$

Proof of (1). Since $\left(1-e_{i}\right) R e_{i}=0$ then $R e_{i}=e_{i} R e_{i}$. Since $e_{i} R e_{i}$ is a division ring then $R e_{i}$ is a minimal left ideal of $R$ so that $R\left(1-e_{i}\right)$ is a maximal left ideal. Thus $J \subseteq \cap R\left(1-e_{i}\right)$. For the other containment, if $r \in \cap R\left(1-e_{i}\right)$ then $r S_{i}=0$ for all $i$ so $r$ (Soc $R)=0$. Then since Soc $R$ is essential in $R_{R}$ it follows that $r \in J$ by [1, Theorem 3.1]. Thus (1) is proved.

(2) The mapping $\alpha: R_{R} \rightarrow \Pi e_{i} R$ defined by $\alpha(r)=\left(e_{i} r\right)_{i \in I}$ is an $R$-monomorphism.

Proof of (2). If $r \in R$ and $r \neq 0$ then since Soc $R$ is essential in $R$ there is $r_{1} \in R$ so that $0 \neq r r_{1} \in \operatorname{Soc} R$. For some $j \in I$ one has $e_{j} r r_{1} \neq 0$ so $e_{j} r \neq 0$ and $\alpha(r) \neq 0$. Thus (2) is proved.

We will identify the module $\Pi S_{i}$ with its image in $\Pi e_{i} R$ under the mapping induced by the inclusions $S_{i} \hookrightarrow e_{i} R$. Since $\alpha(R)$ is injective then $\Pi e_{i} R=\alpha(R) \oplus L$ for some submodule $L_{R}$.

(3) $L \cong \Pi S_{i}$.

Proof of (3). If $L \nsubseteq \Pi \Pi S_{i}$ then there is $\left(x_{i}\right)_{i=1}^{\infty} \in L$ such that $x_{j} \notin$ $S_{j}$ for some $j$. Since $S_{j}=e_{j} R\left(1-e_{j}\right)$ and $x_{j} e_{j}=e_{j} x_{j} e_{j}$ it follows that $x_{j} e_{j} \neq 0$. Then since $x_{i} e_{j}=\delta_{i j} x_{j} e_{j}$ we have $0 \neq\left(x_{i}\right)_{i=1}^{\infty} e_{j}=$ $\alpha\left(x_{j} e_{j}\right) \in L \cap \alpha(R)$ which is a contradiction. Thus (3) is proved.

From (3) and the modular law one has

$$
\Pi S_{i}=\Pi S_{i} \cap(\alpha(R) \oplus L)=\left(\Pi S_{i} \cap \alpha(R)\right) \oplus L .
$$

(4) $\Pi S_{i} \cap \alpha(R)=\alpha(J)$.

Proof of (4). Since $J=\cap R\left(1-e_{i}\right)$ from (1), it is clear that $\alpha(J) \subseteq \Pi S_{i} \cap \alpha(R)$. For the other containment suppose that $r \in R$ and $\alpha(r) \in \Pi S_{i}$. Then for each $i$ one has $e_{i} r=e_{i} r\left(1-e_{i}\right)$ so that $r e_{i}=e_{i} r e_{i}=0$. It follows from (1) that $r \in J$. Thus (4) is proved.

We have $\Pi S_{i}=\alpha(J) \oplus L$ and since $\left(\Pi S_{i}\right) J=0$, it follows from 
(2) that $J^{2}=0$.

(5) The rings $R / J$ and $\Pi e_{i} R e_{i}$ are isomorphic.

Proof of (5). For each $i, e_{i} R=S_{i} \oplus e_{i}, R e_{i}$ as abelian groups. The projections onto the second summands induce an abelian group epimorphism $\pi: \Pi e_{2} R \rightarrow \Pi e_{i} R e_{2}$. Let $\beta=\pi \alpha$ map $R$ into $\Pi e_{i} R e_{i}$. Using $R e_{i}=e_{i} R e_{i}$ one can see that $\beta$ is a ring homomorphism. Since by (3) $L \subseteq \Pi S_{i}$ then $\pi(L)=0$ and since $\Pi e_{i} R=\alpha(R) \oplus L$ it follows that $\operatorname{Im} \pi=\pi \alpha(R)=\operatorname{Im} \beta$ so $\beta$ is an epimorphism. We note that $\operatorname{Ker} \beta=\alpha^{-1}(\operatorname{Ker} \pi)=\alpha^{-1}\left(\Pi S_{i}\right)=J$ by (4). Thus (5) is proved.

From Lemma 2, each $S_{i}$ is a 1-dimensional left vector space over $e_{i} R e_{i}$. The componentwise multiplication $\left(e_{i} r_{i} e_{i}\right)_{i=1}^{\infty}\left(s_{i}\right)_{i=1}^{\infty}=$ $\left(e_{i} r_{i} e_{i} s_{i}\right)_{i=1}^{\infty}$ makes $\Pi S_{i}$ a left $\Pi e_{i} R e_{i}$-module. Since each $S_{i}$ is a left ideal of $R$ and $J S_{i}=0$ then $\Pi S_{i}$ is naturally a left $R / J$-module where the multiplication is given by $(r+J)\left(s_{i}\right)_{i=1}^{\infty}=\left(r s_{i}\right)_{i=1}^{\infty}$. We denote by $D_{i}$ the ring $e_{i} R e_{i}$.

(6) As left $R / J$-modules, $\Pi S_{i}$ is isomorphic to $R / J$.

Proof. In each $S_{i}$ select a nonzero element $x_{i}$. This produces a map $\delta: \Pi S_{i} \rightarrow \Pi D_{i}$ where $\delta\left(s_{i}\right)_{i=1}^{\infty}=\left(d_{i}\right)_{i=1}$ when $s_{i}=d_{i} x_{i}$. The mapping $\delta$ is clearly a $\Pi D_{i}$-isomorphism. The mapping $\beta$ of (5) induces a ring isomorphism $\bar{\beta}: R / J \rightarrow \Pi D_{i}$. One checks that if $\bar{r}=r+J$ for $r \in R$ and $s \in \Pi S_{i}$ then $\delta(\bar{r} s)=\beta(r) \delta(s)$ so that if we identify $R / J$ and $\Pi D_{i}$ via $\bar{\beta}$ then $\delta$ yields the desired isomorphism. Thus (6) is proved.

Since $J^{2}=0$ then $J$ is a left $R / J$-module.

(7) The restriction of $\alpha$ to $J$ is an $R / J$-monomorphism from ${ }_{R / J} J$ into $\Pi S_{i}$.

Proof of (7). If $s \in \Pi S_{i}$ and $\bar{r}=r+J$ for $r \in R$ then one always has $\bar{r} s=\bar{\beta}(\bar{r}) \cdot s$ where denotes componentwise multiplication, since $\bar{\beta}(\bar{r}) \cdot s=\beta(r) \cdot s=\left(e_{i} r e_{i}\right) \cdot\left(s_{i}\right)=\left(e_{i} r e_{i} s_{i}\right)=\left(r e_{i} s_{i}\right)=\left(r s_{i}\right)=r\left(s_{i}\right)=\bar{r} s$. Let $j$ belong to $J$. Then $\alpha(\bar{r} j)=\alpha(r j)=\left(e_{i} r_{i}\right)_{i=1}^{\infty} j=\left(e_{i} r e_{i} j+e_{i} r\left(1-e_{i}\right) j\right)_{i=1}^{\infty}=$ $\left(e_{i} r e_{i} j\right)_{i=1}^{\infty}=\left(e_{i} r e_{i}\right)_{i=1}^{\infty} . \quad\left(e_{i} j\right)_{i=1}^{\infty}=\bar{\beta}(r) \cdot \alpha(j)=\bar{r} \alpha(j)$. Thus (7) is proved.

(8) The mapping $\bar{\beta}^{-1} \delta \alpha$ is an essential embedding of $J$ into $R / J$ as left $R / J$-modules.

Proof of (8). From (6) and (7), $\bar{\beta}^{-1} \delta \alpha$ is an $R / J$-embedding of $J$ into $R / J$. To show that $\bar{\beta}^{-1} \delta \alpha(J)$ is essential in $R / J$ is equivalent to showing that $\delta \alpha(J)$ is an essential left ideal of $\Pi D_{i}$. It suffices to show that for each $j$ the idempotent $E_{j}$ where $E_{j}=\left(\delta_{i j}\right)_{i=1}^{\infty}$ belongs to $\delta \alpha(J)$. If $x_{i} \in S_{i}$ then for each $k$ one has $x_{i} e_{k}=0$ so that $\Sigma S_{i} \subseteq$ $J$. Then with the elements $x_{i} \in S_{i}$ chosen in (6) one has $\delta \alpha\left(x_{j}\right) \in$ $\delta \alpha(J)$ and $\delta \alpha\left(x_{j}\right)=\delta\left(e_{i} x_{j}\right)_{i=1}^{\infty}=\delta\left(\delta_{i j} x_{j}\right)={ }_{i=1}^{\infty} E_{j}$. Thus (8) is proved. 
Consider the bimodule ${ }_{R / J} J_{R / J}$. The right hand action of elements of $R / J$ on $J$ produces a ring homomorphism $\gamma: R / J \rightarrow \operatorname{End}\left({ }_{R / J} J\right)$ whose kernel is $\{r+J \mid J r=0\}$.

(9) If $r$ belongs to $R$ then $J r=0$ if and only if Supp $r=$ $\left\{i \in I \mid r e_{i} \neq 0\right\}$ is finite.

Proof of (9). Suppose that Supp $r$ is finite. Since $r_{1}=r-$ $\sum_{i \in \operatorname{Supp}}{ }_{r} r_{i}$ left annihilates all the $e_{i}$ then by (1) $r_{1}$ belongs to $J$ and hence $J r_{1}=0$ since $J^{2}=0$. But clearly $J \sum_{i \in \operatorname{Supp}_{r} r e_{i}=}$ $J \sum_{i \in \operatorname{Supp} r} e_{i} r e_{i}=0$. Thus $J r=0$.

Suppose that $J r=0$ and that Supp $r$ is infinite. From Lemma 3 there is a central idempotent $f$ of $R$ so that if $I_{1}=\left\{i \in I \mid e_{i} \in f R\right\}$ then Supp $r \Delta I_{1}$ is finite. Replacing $R$ by $f R$ we can assume that Supp $r$ is cofinite in $I$. Let $r_{2}=r+\sum_{i \notin \operatorname{Supp} r} e_{i}$. Since $J\left(\Sigma e_{i}\right)=0$ then $J r_{2}=0$. But for all $i$ in $I, r_{2} e_{i} \neq 0$ so $\beta\left(r_{2}\right)$ is a unit of $\Pi D_{i}$. Then there is an element $t$ of $R$ so that $1-r_{2} t \in J$ and hence $J=$ $J\left(1-r_{2} t\right) \subseteq J^{2}=0$, a contradiction. Thus (9) is proved.

It follows from (9) that $\operatorname{Ker} \gamma=\{\bar{r} \mid J r=0\}=\left\{\bar{r} \mid r e_{i}=e_{i} r e_{i}=0\right.$ a.e. $\}=\left\{\bar{r} \mid \bar{\beta}(r) \in \operatorname{Soc} \Pi D_{i}\right\}=\operatorname{Soc}(R / J)$. Let $D=\Pi D_{i}=\Pi e_{i} R e_{i}$. Then $\gamma$ induces a ring monomorphism from $D /$ Soc $D$ into End $\left(_{R / J} J\right)$. Since as a left $R / J$-module $J$ is isomorphic to an essential left ideal of $R / J$ by (8), then End $\left(_{R / J} J\right) \simeq R / J$ because $R / J$ is a left self-injective regular ring (in fact, a product of division rings). It follows that $\gamma$ induces a ring monomorphism from $D / \operatorname{Soc} D$ into $D$. We then arrive at a contradiction from the following two facts.

(10) If $G$ is a set of nonzero orthogonal idempotents of $D=\Pi D_{i}$ then $|G| \leqq \boldsymbol{\aleph}_{0}$.

(11) The ring $D /$ Soc $D$ has a set of orthogonal idempotents of cardinality $c$.

Proof of (10). For each $i=1,2, \cdots$ let $\varepsilon_{i}$ be the sequence of $D$ with $i$ th slot $e_{i}$ and zero elsewhere. If $g_{1}$ and $g_{2}$ belong to $G$ and $\varepsilon_{i} g_{1}=\varepsilon_{i}$ and $\varepsilon_{i} g_{2}=\varepsilon_{i}$ then $g_{1}=g_{2}$ since otherwise we have $\varepsilon_{i}^{2}=0$. It follows that if we let $E=\left\{\varepsilon_{i} \mid \varepsilon_{i} g=\varepsilon_{i}\right.$ for some $g$ in $\left.G\right\}$ then the mapping from $E$ to $G$ which maps $\varepsilon_{i}$ to $g$ if $\varepsilon_{i} g=\varepsilon_{i}$ is well-defined and it is clearly a surjection. It follows that $|G| \leqq|E| \leqq \aleph_{0}$.

Proof of (11). The set $N$ of natural numbers has a set $\mathscr{A}$ of $c$ subsets of $N$, each of cardinality $\aleph_{0}$, any two of which have finite intersection. (Match $N$ with the set of rational numbers and choose for each real number a strictly increasing sequence of rational numbers converging to it.) For each subset $X$ of $N$ let $e_{X}$ be the idempotent of $D$ such that $e_{X}(i)=e_{i}$ if $i$ belongs to $X$ and 
$e_{X}(i)=0$ otherwise. The set $\mathscr{A}=\left\{e_{X}+\operatorname{Soc} D \mid X \in \mathscr{A}\right\}$ is a set of pairwise orthogonal idempotents of $D /$ Soc $D$. Since $X \Delta Y$ is infinite when $X$ and $Y$ are distinct members of $\mathscr{A}$ then $e_{X}+\operatorname{Soc} D \neq e_{Y}+$ Soc $D$. It follows that $\mathscr{A}$ has cardinality $c$.

Thus (10) and (11) hold and Theorem 5 is proved.

CoRollary. Let $R$ be a basic right q-ring which has no projective minimal right ideals and has no loops. Then $R$ is a finite product of chain q-rings whose corners are right q-rings with no noncentral idempotents.

Proof. It follows from Theorem 5 that $\mathscr{A}(R)$ is a finite set all of whose members are chains. If $\mathscr{C}(R)$ is the union of the sets in $\mathscr{A}(R)$ then $\Sigma \mathscr{C}(R)$ is injective so there is an idempotent $g$ of $R$ such that $R_{R}=g R+\Sigma \mathscr{C}(R)$. If the chains are denoted $\vec{E}_{i}$ or $\rightarrow E_{\imath 1} \rightarrow E_{\imath 2} \rightarrow \cdots \rightarrow E_{\imath l_{\imath}}$ for $1 \leqq i \leqq m$ then $g R \rightarrow E_{i 1}$ for each $i$. As in Proposition 6 we can find orthogonal idempotents $g_{2}, 1 \leqq i \leqq$ $m$ so that $g=\sum_{i=1}^{m} g_{i}$ and $g_{2} R \rightarrow E_{j_{1}}$ if and only if $i=j$. If $\bar{g}_{i}$ is an idempotent such that $\bar{g}_{i} R=g_{i} R \oplus \sum_{j=1}^{l_{i}} E_{\imath j}$ then $\bar{g}_{i}$ is central in $R$. As a ring $g_{i} R$ is a chain right $q$-ring such that the corner $g_{i} R=$ $g_{i} R g_{i}$ is a right $q$-ring with $\mathscr{A}\left(g_{2} R\right)=\varnothing$. It follows from Lemma 1 that each idempotent of $g_{2} R$ is central.

Proposition 9. If $R$ is a right q-ring with no projective minimal right ideals all of whose idempotents are central then $R \simeq$ $Z \times L$ where $Z$ is a right q-ring with no primitive idempotent and $L$ is a product of local right q-rings none of which is a division ring.

Proof. Let $\left\{e_{\imath} \mid i \in I\right\}$ be the set of primitive idempotents of $R$. As in the proof of Proposition 7 there is an idempotent $g$ of $R$ so that $g R$ is ring-isomorphic to the product of local rings $L=\Pi e_{i} R$, in such a way that $e_{i} R \subseteq g R$ corresponds to its usual image in $\Pi e_{i} R$. Clearly, $(1-g) R$ has no primitive idempotent.

We note that local rings in the product $L$ which are division rings would correspond to projective minimal right ideals of $R$.

Proposition 10. Let $R$ be a chain right q-ring without projective minimal right ideals and with corner $g R$ a ring with all idempotents central. Then $R \simeq R_{1} \times L$ where $R_{1}$ is a chain right q-ring with corner $Z$ a ring with no primitive idempotents and $L$ is a product of local right q-rings none of which is a division ring.

Proof. By Proposition 9, $g R=g_{1} R \oplus g_{2} R$ where $Z=g_{1} R$ has 
no primitive idempotent and $g_{2} R \simeq L$ is a product of local right $q$ rings none of which is a division ring. The chain of $R$ is associated with $Z$ and not with $L$. This follows from Proposition 5 and the fact that if $L$ is a product of local rings which are not division rings then there is no simple, injective right $L$-module which is not embeddable in $L$. For suppose that $L=\Pi L_{i}$ where each $L_{i}$ is a local right $q$-ring so that $J_{i} \neq 0$ where $J_{\imath}$ is the Jacobson radical of $L_{i}$. Suppose $L / M$ is simple, injective $L$-module and is not embeddable in $L$. Then the maximal right ideal $M$ of $L$ is essential and therefore $M$ is an ideal of $L$. Choose $u \in L$ so that $u=\left(u_{i}\right)$ where for each $i, u_{i} \in J_{i}$ and $u_{i} \neq 0$. The right annihilator $u^{r}$ of $u$ in $L$ is contained in the radical $\Pi J_{i}$ of $L$ so that in particular $u^{r} \subseteq M$. Thus the mapping $u a \mapsto a+M$ from $u L$ to $L / M$ is a well-defined epimorphism. Since $L / M$ is injective there is an element $x \in L / M$ so that for each $a \in L, a+M=x(u a)$. But $u \in \Pi J_{i} \cong M$ so that $x u=$ 0 and we have a contradiction.

We can summarize all of the structure theorems of the paper in the following way.

THEOREM 6. A right q-ring is isomorphic to a finite product of rings of the following kinds:

(1) Semisimple artinian ring.

(2) Loop q-ring: $H(l, D)$.

(3) $\Pi D_{i}$-chain q-ring: $C\left(\Pi D_{i}, M, l\right)$ where the corner $\Pi D_{i}$ is an infinite product of division rings.

(4) Z-chain q-ring: $C(Z, M, l)$ where the corner $Z$ is a right q-ring with no primitive idempotent.

(5) A product of local right q-rings none of which is a division ring.

Final remarks. The further study of $q$-rings would examine the structure of the local ones and the ones which have no primitive idempotent. The latter clearly have zero right socle and for both kinds, all idempotents are central so that one would expect the investigation of them to require methods very different from those of the present paper.

With regard to the symmetry question for the $q$-ring condition, it is easy to see that a chain right $q$-ring (of length $\geqq 1$ ) is not left self-injective so that a right $q$-ring need not be also a left $q$ ring. For consider $R=C(A, M, 1)$ and let $E_{1}$ and $E_{2}$ be the idempotent matrices with zero entries except for entries of 1 in the $(1,1)$ and $(2,2)$ positions respectively. It is easy to see that the obvious correspondence between $S=E_{2} R E_{1}$ and $R E_{2}$ where $\left(\begin{array}{ll}0 & 0 \\ x & 0\end{array}\right)$ 
corresponds to $\left(\begin{array}{ll}0 & 0 \\ 0 & x\end{array}\right)$ is an isomorphism of left $R$-modules. If $R$ were left self-injective then by Baer's criterion the isomorphism from $S$ to $R E_{2}$ could be realized as a right multiplication by some element of $R E_{2}$, but $S R E_{2}=0$. One might rephrase the question thus: Is every right $q$-ring with no chain of length $\geqq 0$ also a left $q$-ring? [Cf. 2, Remark 2.14.] With regard to this symmetry question one would like to know whether there is a local, right selfinjective duo ring which is not left self-injective.

\section{REFERENCES}

1. C. Faith and Y. Utumi, Quasi-injective modules and their endomorphism rings, Arch. Math., 15 (1964), 166-174.

2. D. A. Hill, Semiperfect q-Rings, Math. Ann., 200 (1973), 113-121.

3. G. Ivanov, Non-local rings whose ideals are all quasi-injective, Bull. Austral. Math. Soc., 6 (1972), 45-52.

4. - Non-local rings whose ideals are all quasi-injective: Addendum, Bull. Austral. Math. Soc., 12 (1975), 159-160.

5. N. Jacobson, Structure of Rings, AMS Colloquium Publ., Vol. 37, Providence, R. I., 1964.

6. R. E. Johnson and E. T. Wong, Quasi-injective modules and irreducible rings, J. London Math. Soc., 36 (1961), 260-268.

Received August 2, 1977 and in revised form July 31, 1978.

The University of North Carolina

GREENSBORO, NC 27412 



\section{PACIFIC JOURNAL OF MATHEMATICS}

\section{EDITORS}

DoNALD BABBITT (Managing Editor)

University of California

Los Angeles, California 90024

HUGo Rossi

University of Utah

Salt Lake City, UT 84112

C. C. MOORE and ANDREW OGG

University of California

Berkeley, CA 94720
J. DUGUNDJI

Department of Mathematics University of Southern Californı Los Angeles, California 90007

R. Finn and J. Milgram Stanford University Stanford, California 94305

\section{ASSOCIATE EDITORS}

E. F. BECKENBACH

B. H. Neumann
F. WOLF

K. YOSHIDA

\section{SUPPORTING INSTITUTIONS}

UNIVERSITY OF BRITISH COLUMBIA CALIFORNIA INSTITUTE OF TECHNOLOGY UNIVERSITY OF CALIFORNIA MONTANA STATE UNIVERSITY UNIVERSITY OF NEVADA, RENO NEW MEXICO STATE UNIVERSITY OREGON STATE UNIVERSITY UNIVERSITY OF OREGON
UNIVERSITY OF SOUTHERN CALIFORNIA STANFORD UNIVERSITY UNIVERSITY OF HAWAII UNIVERSITY OF TOKYO UNIVERSITY OF UTAH WASHINGTON STATE UNIVERSITY UNIVERSITY OF WASHINGTON 


\section{Pacific Journal of Mathematics}

Vol. 82, No. $1 \quad$ January, 1979

Werner Bäni, Subspaces of positive definite inner product spaces of countable dimension ...................................... 1

Marilyn Breen, The dimension of the kernel of a planar set..............

Kenneth Alfred Byrd, Right self-injective rings whose essential right ideals

are two-sided

Patrick Cousot and Radhia Cousot, Constructive versions of Tarski's fixed

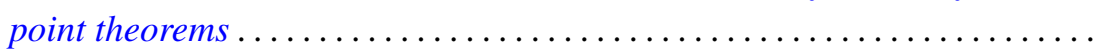

Ralph S. Freese, William A. Lampe and Walter Fuller Taylor, Congruence lattices of algebras of fixed similarity type. $I \ldots \ldots \ldots \ldots \ldots \ldots \ldots$

Cameron Gordon and Richard A. Litherland, On a theorem of Murasugi .....

Mauricio A. Gutiérrez, Concordance and homotopy. I. Fundamental

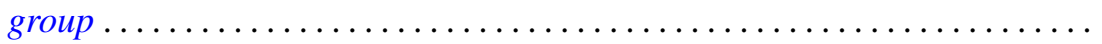

Richard I. Hartley, Metabelian representations of knot groups .............

Ted Hurley, Intersections of terms of polycentral series of free groups and free

Lie algebras ........................................

Roy Andrew Johnson, Some relationships between measures ............ 117

Oldřich Kowalski, On unitary automorphisms of solvable Lie algebras .......

Kee Yuen Lam, $K O$-equivalences and existence of nonsingular bilinear

maps...................................................

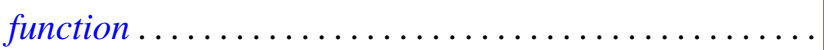

Robert A. Messer and Alden H. Wright, Embedding open 3-manifolds in compact 3-manifolds ............................

Gerald Ira Myerson, A combinatorial problem in finite fields. I . .

James Nelson, Jr. and Mohan S. Putcha, Word equations in a band of paths.

Baburao Govindrao Pachpatte and S. M. Singare, Discrete generalized Gronwall inequalities in three independent variables . .

William Lindall Paschke and Norberto Salinas, $C^{*}$-algebras associated with free products of groups ........................

Bruce Reznick, Banach spaces with polynomial norms ....

David Rusin, What is the probability that two elements of a finite group

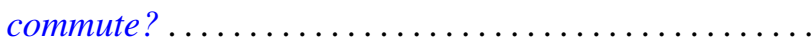

M. Shafii-Mousavi and Zbigniew Zielezny, On hypoelliptic differential operators of constant strength ...

Joseph Gail Stampfli, On selfadjoint derivation ranges .... . . .

Robert Charles Thompson, The case of equality in the matrix-valued triangle

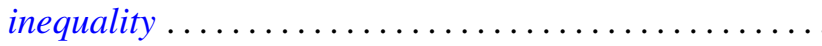

Marie Angela Vitulli, The obstruction of the formal moduli space in the negatively graded case. 